\title{
Antropometria e composição corporal de recém-nascidos pré-termo na idade gestacional e no peso equivalente ao termo
}

\author{
Anthropometry and body composition \\ of preemies at term age and \\ term age weight
}

Letícia Duarte VILLELA ${ }^{1}$

Fernanda Valente MENDES SOARES'

Andrea Dunshee de ABRANCHES ${ }^{1}$

Saint-Clair GOMES JUNIOR ${ }^{1}$

Maria Dalva Barbosa Baker MÉIO ${ }^{1}$

Maria Elisabeth Lopes MOREIRA ${ }^{1}$

\section{RES U M O}

\section{Objetivo}

Analisar o crescimento e a composição corporal de recém-nascidos pré-termo na idade gestacional corrigida de termo e ao alcançarem um peso entre 3,0 e 3,5 kg.

\section{Métodos}

Estudo longitudinal, realizado no Instituto Fernandes Figueira, Rio de Janeiro, com 39 recém-nascidos pré-termo e que apresentaram muito baixo peso ao nascer. Medidas antropométricas e água corporal total foram avaliadas no primeiro, no sétimo e no dia da recuperação do peso de nascimento, na idade gestacional corrigida do termo e em torno de três semanas de idade gestacional corrigida (correspondente ao tempo de vida para alcançar um peso entre 3,0 e 3,5 kg). O grupo de referência foi constituído por 32 recém-nascidos a termo, adequados para a idade gestacional, avaliados no segundo dia de vida. Considerou-se restrição de crescimento o escore-Z menor do que -2 para peso, comprimento e perímetro cefálico.

\footnotetext{
1 Fundação Oswaldo Cruz, Instituto Nacional de Saúde da Mulher, da Criança e do Adolescente Fernandes Figueira, Unidade de Pesquisa Clínica. Av. Rui Barbosa, 716, Bloco 2, Flamengo, 22250-020, Rio de Janeiro, RJ, Brasil. Correspondência para/Correspondence to: MEL MOREIRA. E-mail: <bebeth@iff.fiocruz.br>.

Apoio: Fundação de Amparo à Pesquisa do Estado do Rio de Janeiro (Processo no 102.873/2008).
} 


\section{Resultados}

Na idade de termo, $71,8 \%$ dos recém-nascidos pré-termo apresentaram restrição do crescimento para peso, $61,5 \%$ para comprimento e 25,6\% para perímetro cefálico. Com três semanas de idade gestacional corrigida, esses recém-nascidos apresentaram a prega cutânea tricipital e a circunferência abdominal estatisticamente maiores que o grupo de referência enquanto o comprimento e a porcentagem de água corporal total foram menores.

\section{Conclusão}

Os recém-nascidos pré-termo apresentaram perfil antropométrico e de água corporal diferente dos recém-nascidos a termo, sugerindo acúmulo de gordura. Houve recuperação do crescimento entre a idade de termo e três semanas de idade corrigida, sendo mais evidente esse crescimento em relação ao perímetro cefálico e peso.

Palavras-chave: Antropometria. Composição corporal. Prematuro. Recém-nascido.

\section{A B S T R A C T}

\section{Objective}

To analyze growth and body composition of preemies at term gestational age and when they reach a weight of 3.0 to $3.5 \mathrm{~kg}$.

\section{Methods}

This longitudinal study was conducted at Instituto Fernandes Figueira, Rio de Janeiro, and included 39 preemies with very low birth weight. Anthropometric measurements and bioelectrical impedance analysis were performed in 5 occasions: days 1 and 7 after birth; at birth weight recovery; at term-corrected gestational age; and at around three weeks of corrected gestational age (age needed to reach a weight of 3.0 to $3.5 \mathrm{~kg}$ ). A reference group comprising 32 term newborns, appropriate for gestational age, was evaluated at day 2 after birth. Growth restriction was defined as a Z-score below -2 for weight, length, and head circumference.

\section{Results}

At term age, $71.8 \%$ of the preemies presented growth restriction for weight, $61.5 \%$ for length, and $25.6 \%$ for head circumference. When the preemies reached three weeks of corrected gestational age, triceps skinfold thickness and abdominal circumference were greater than those of the reference group, and length and percent of total body water were lower.

\section{Conclusion}

The anthropometric profile and total body water of preemies were different from those of term newborns, suggesting fat deposition. There was catch up growth between term age and three weeks of corrected gestational age, which was more evident for head circumference and weight.

Keywords: Anthropometry. Body composition. Infant, premature. Infant, newborn.

\section{N T R O D U Ç Ã O}

Os recém-nascidos com idades gestacionais e pesos cada vez menores admitidos nas unidades de terapia intensiva e a preocupação com o desenvolvimento nos primeiros anos de vida fazem com que o crescimento pós-natal seja um tema em foco na neonatologia ${ }^{1-3}$.

O crescimento pós-natal de Recém-Nascidos Pré-Termo (RNPT) deve ser semelhante ao que ocorre intraútero, tanto na velocidade de crescimento como na composição corporal4-6. Apesar dessa recomendação, a restrição do crescimento pós-natal é uma realidade mundial, e estudos atuais sugerem uma nutrição precoce e agressiva do ponto de vista proteico-calórico como tentativa de evitá-la $a^{7-11}$. Entretanto, existe a preocupação com o crescimento acelerado no período pós-natal, que pode estar associado com o desenvolvimento da síndrome metabólica e doença cardiovascular na vida adulta ${ }^{12-16}$. Segundo Barker ${ }^{17}$, a alteração permanente no 
metabolismo corporal pode ter origem nas fases iniciais do desenvolvimento.

Em 2012, foi realizada uma metanálise que incluiu oito estudos e analisou a composição corporal de 733 RNPT e termo. Os autores verificaram que os RNPT alcançaram a idade gestacional corrigida do termo com maior porcentagem de gordura corporal total e significativa redução da massa livre de gordura ${ }^{18}$. O melhor entendimento sobre crescimento pós-natal, restrição e composição corporal pode ser um guia no tratamento nutricional dos RNPT, mas ainda são poucos os estudos que abordam o tema, principalmente na população brasileira.

O objetivo do estudo foi analisar o crescimento e a composição corporal de RNPT na idade gestacional corrigida de termo e com três semanas de idade gestacional corrigida (correspondente ao tempo de vida para os RNPT alcançarem um peso entre 3,0 e 3,5 kg).

\section{M É T O D O S}

Trata-se de um estudo longitudinal realizado com RNPT de muito baixo peso ao nascer, admitidos na Unidade de Terapia Intensiva Neonatal do Instituto Nacional em Saúde da Mulher, da Criança e do Adolescente Fernandes Figueira/ Fundação Oswaldo Cruz (Fiocruz) em 2010. Os recém-nascidos a termo, adequados para a idade gestacional, foram incluídos como grupo de referência.

Foi considerado RNPT de muito baixo peso aquele com menos que 37 semanas de idade gestacional e peso menor que $1500 \mathrm{~g}$ ao nascimento e recém-nascido a termo aquele com a idade gestacional ao nascimento entre 37 e 41 semanas.

Para o cálculo da idade gestacional ao nascimento, foi utilizada a data da última menstruação materna e, em caso desse dado não estar disponível, a ultrassonografia de primeiro trimestre ou a avaliação de Ballard, nessa ordem ${ }^{19}$. A idade gestacional foi corrigida no decorrer do estudo pelo acréscimo de semanas de vida pós-natal, e foi considerada idade gestacional corrigida de termo entre 37 e 41 semanas.

Foram excluídos os recém-nascidos com malformação congênita, síndrome genética, infecção congênita do grupo Toxoplasmosis, Rubella Cytomegalo Vírus, and Herpes (TORCH, Toxoplasmose, Rubéola, Citomegalovírus e Herpes), filhos de mães com vírus da imunodeficiência humana (HIV positivas) e filhos de mães diabéticas.

A Figura 1 apresenta o fluxo de seleção dos participantes, de acordo com os critérios de elegibilidade adotados. As variáveis estudadas foram: peso, comprimento, perímetro cefálico, circunferência abdominal, prega cutânea tricipital e porcentagem de água corporal total. Também foi analisada a razão da porcentagem de água corporal total e prega cutânea tricipital, com o objetivo de verificar se a diminuição de água corporal total (\%) estava relacionada ao ganho na prega cutânea tricipital.

Os recém-nascidos pré-termo foram avaliados em 5 momentos: no primeiro dia de vida, no sétimo dia de vida, no dia da recuperação do peso de nascimento, na idade gestacional corrigida de termo e posteriormente em torno de três semanas de idade gestacional corrigida (correspondente ao tempo de vida para alcançar um peso entre 3,0 e 3,5 kg, semelhante ao peso do recém-nascido a termo). Os recém-nascidos a termo foram avaliados no segundo dia, após 36 horas de vida, período posterior à maior perda de água do compartimento extracelular ${ }^{20}$.

Considerando uma confiança de $95 \%$, um poder de $80 \%$ e as estimativas do estudo-piloto e de outros estudos publicados, calculou-se uma amostra de 31 recém-nascidos, levando-se em conta as diferenças na água corporal total entre os RNPT e termo ${ }^{21-23 .}$

Para avaliação antropométrica, a aferição do peso foi feita com balança digital Filizola ${ }^{\circledR}$ (São Paulo [SP]) com sensibilidade de $5 \mathrm{~g}$. O com- 


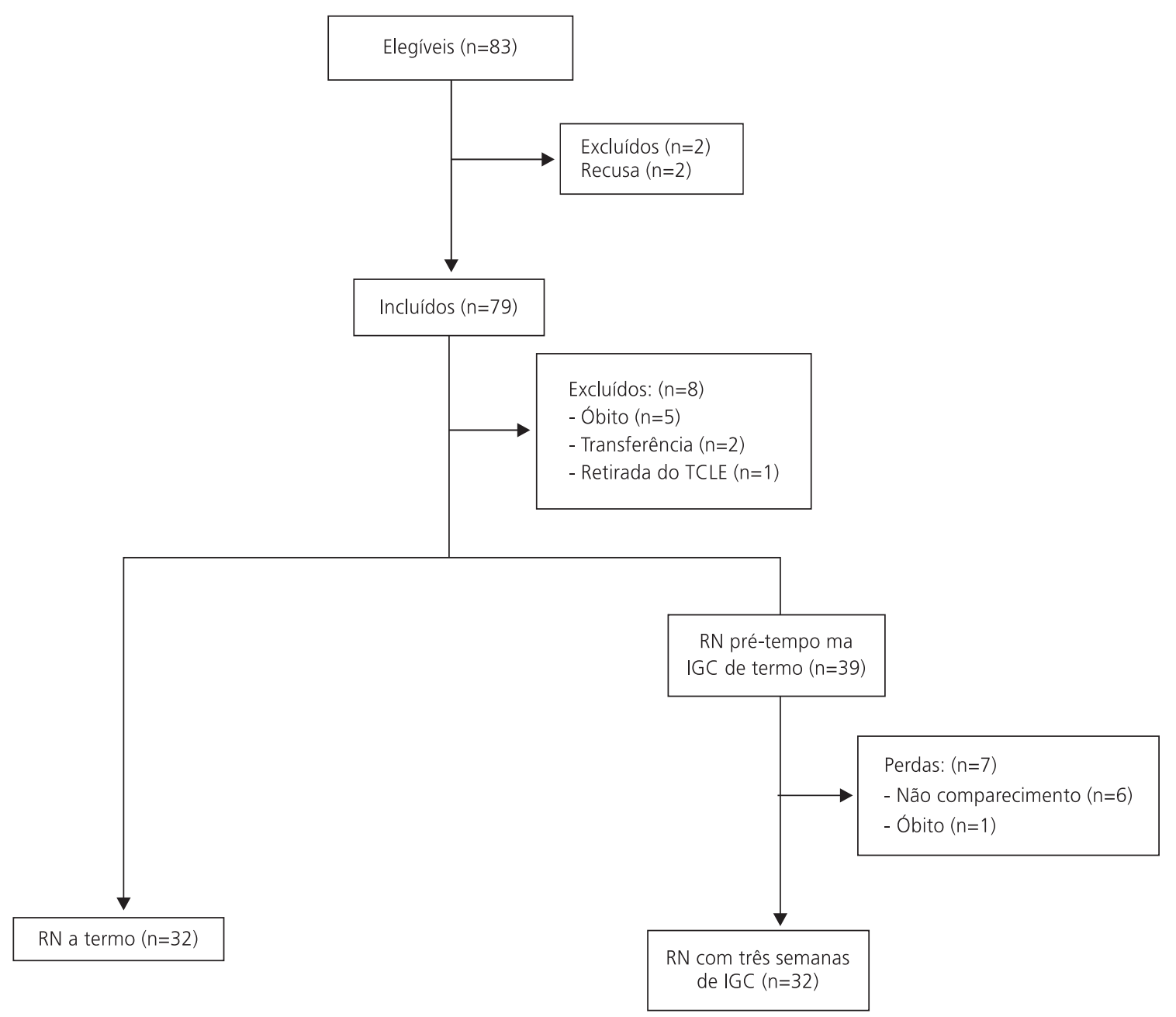

Figura 1. Fluxo de seleção dos participantes de acordo com os critérios de elegibilidade. Instituto Nacional de Saúde da Mulher, da Criança e do Adolescente Fernandes Figueira, Rio de Janeiro (RJ), 2010.

Nota: TCLE: Terno de Consetimento Livre e Esclarecido; RN: Recém-Nacidos; IGC: Idade Gestacional Corrigida.

primento foi obtido por uma régua antropométrica - idealizada e confeccionada na oficina de precisão da Universidade de São Paulo de Ribeirão Preto -, que apresenta lâminas de acrílico unidas de forma retangular, permitindo encaixe na superfície da incubadora ou berço24. A medida do perímetro cefálico foi obtida por meio de uma fita milimetrada inextensível, que foi ajustada à cabeça do recém-nascido, passando anteriormente pela região supraorbitária e posteriormente pela proeminência occipital, a fim de se registrar o maior perímetro obtido ${ }^{25}$. A circunferência abdominal foi realizada com a fita métrica inextensível com recém-nascido em decúbito dorsal, na linha da cintura, no ponto médio entre a última costela e a crista ilíaca ${ }^{26}$. A prega cutânea tricipital foi obtida no ponto médio entre o acrômio e o olecrânio do braço esquerdo por meio do plicômetro tipo Cescorf ${ }^{\circledR}$ (Cescorf Equipamentos Esportivos Ltda., Porto Alegre, Rio Grande do Sul) ${ }^{27}$.

O cálculo do escore-Z do peso, comprimento e perímetro cefálico para a idade gesta- 
cional foi realizado por meio da curva de crescimento neonatal publicada em 2013 por Fenton \& $\mathrm{Kim}^{28}$. Essa curva permite o cálculo do escore-Z para a idade gestacional e sexo, desde 23 semanas a 50 semanas de idade gestacional corrigida e, portanto, é adequada para avaliar o crescimento longitudinalmente.

A bioimpedância elétrica foi utilizada para avaliar a porcentagem de água corporal total dos recém-nascidos por meio do valor da resistência. Essa medida foi realizada por meio do equipamento RJL System (RJL System Inc., Clinton Township, Michigan, Estados Unidos, aparelho Quantum BIA 101Q), que é de baixo custo, não invasivo, portátil e que possibilita o exame na incubadora ou à beira do leito. O cálculo da água corporal total foi obtido a partir da equação proposta por Tang et al. ${ }^{23}$

Os dados foram inseridos e analisados no software Statistical Package for the Social Sciences (SPSS Inc., Chicago, Illinóis, Estados Unidos) 21.0. Foram utilizados a Análise de Variância (Anova) para medidas repetidas para verificação de diferenças dos RNPT nos diferentes momentos e o teste $t$ para comparação entre os RNPT e termo. Para o tratamento estatístico da razão da porcentagem de água corporal total e prega cutânea tricipital, foi utilizado o teste de Mann-Whitney, pois essas variáveis não apresentaram uma distribuição normal. Para todas as análises, considerou-se um nível de significância de 0,05.

O estudo foi aprovado pelo Comitê de Ética em Pesquisa do Instituto Fernandes Figueira (CAAE 0292.0.008.000-05), e foi obtido o Termo de Consentimento Livre e Esclarecido assinado pelos responsáveis antes do início do estudo.

\section{RES U L T A D O S}

Foram elegíveis para o estudo 83 recém-nascidos. Conforme demonstrado no diagrama de fluxo (Figura 1), no momento da comparação por idade, 32 pertenciam ao grupo dos recém-nascidos a termo e 39 ao dos pré-termo. Ao longo do seguimento dos RNPT, houve 7 perdas: 1 por óbito e 6 não compareceram ao ambulatório para avaliação das medidas com três semanas de idade gestacional corrigida.

Os recém-nascidos pré-termo apresentaram média \pm desvio-padrão do peso ao nascer de $1095 \mathrm{~g} \pm 210$ e da idade gestacional de $29,7 \pm 2,2$. No momento do nascimento, quatro RNPT foram classificados como pequenos para a idade gestacional, considerando o peso menor que o percentil 3 da curva de crescimento de Fenton $^{28}$. Os demais foram classificados como adequados para a idade gestacional. Os RNPT apresentaram, com três semanas de idade gestacional corrigida, a média \pm desvio-padrão do peso (3122 g \pm 266 ) semelhante à dos recém-nascidos a termo, de $3174 \mathrm{~g} \pm 226$ (Tabela 1). Ao nascimento, a média \pm desvio-padrão do perímetro cefálico e do comprimento ao nascer dos recém-nascidos a termo foi de $34,6 \mathrm{~cm} \pm 0,9$ e de 49,6 $\mathrm{cm} \pm 1,3$, respectivamente.

$\mathrm{Na}$ idade gestacional corrigida do termo, $71,8,61,5$ e $25,6 \%$ dos RNPT apresentaram o escore- $Z$ para a idade gestacional menor que -2 para o peso, comprimento e perímetro cefálico, respectivamente.

Na análise longitudinal dos RNPT, verifica-se que houve uma redução do escore-Z do peso e comprimento do nascimento até a idade gestacional corrigida de termo e um aumento significativo do escore-Z do perímetro cefálico entre o sétimo dia de vida e a idade gestacional corrigida de termo $(p=0,043)$. A recuperação do peso e do perímetro cefálico foi mais significativa no período compreendido entre a idade gestacional corrigida de termo e três semanas de idade gestacional corrigida (Figura 2). Nesse mesmo período, os RNPT apresentaram um aumento de $64 \%$ da prega cutânea tricipital e diminuição da porcentagem de água corporal total (Tabela 1).

Ao comparar os resultados encontrados com o grupo de referência, verifica-se que, na idade gestacional corrigida de termo, os RNPT apresentaram valores das variáveis antropométricas significativamente inferiores $(p<0,001)$. Ao 
Tabela 1. Evolução das medidas antropométricas e porcentagem de água corporal total dos recém-nascidos pré-termo. Instituto Nacional de Saúde da Mulher, da Criança e do Adolescente Fernandes Figueira, Rio de Janeiro (RJ), 2010.

\begin{tabular}{|c|c|c|c|c|c|c|c|c|c|c|}
\hline \multirow{2}{*}{ Variáveis } & \multicolumn{2}{|c|}{ D1 } & \multicolumn{2}{|c|}{ D7 } & \multicolumn{2}{|c|}{ PN } & \multicolumn{2}{|c|}{ IGC termo } & \multicolumn{2}{|c|}{3 semanas de IGC } \\
\hline & $M$ & $\mathrm{DP}$ & $M$ & $\mathrm{DP}$ & $M$ & DP & $\mathrm{M}$ & $\mathrm{DP}$ & $M$ & $\mathrm{DP}$ \\
\hline Peso (g) & 1095 & 210 & 1022 & 252 & 1140 & 234 & 2154 & 537 & 3122 & 266 \\
\hline Comprimento $(\mathrm{cm})$ & 36,7 & 2,7 & 37,1 & 2,7 & 38,4 & 2,4 & 44,1 & 2,7 & 48,6 & 1,4 \\
\hline $\mathrm{PC}(\mathrm{cm})$ & 26,1 & 1,8 & 26,0 & 2,2 & 27,5 & 1,8 & 32,6 & 1,7 & 35,6 & 1,4 \\
\hline $\mathrm{CA}(\mathrm{cm})$ & 22,1 & 1,8 & 22,0 & 2,0 & 23,7 & 1,8 & 29,7 & 2,6 & 33,5 & 2,3 \\
\hline $\mathrm{PCT}(\mathrm{mm})$ & 0,5 & 0,4 & 1,0 & 0,7 & 1,2 & 0,7 & 2,8 & 1,4 & 4,6 & 1,0 \\
\hline $\mathrm{ACT}(\%)$ & 78,9 & 2,4 & 75,6 & 2,0 & 74,9 & 2,4 & 70,5 & 3,5 & 65,1 & 1,6 \\
\hline
\end{tabular}

Nota: D1: Primeiro dia de vida; D7: Sétimo dia de vida; PN: Dia de recuperação do peso de nascimento; M: Média; DP: Desvio-Padrão; IGC: Idade Gestacional Corrigida; PC: Perímetro Cefálico; CA: Circunferência Abdominal; PCT: Prega Cutânea Tricipital; ACT: Água Corporal Total.
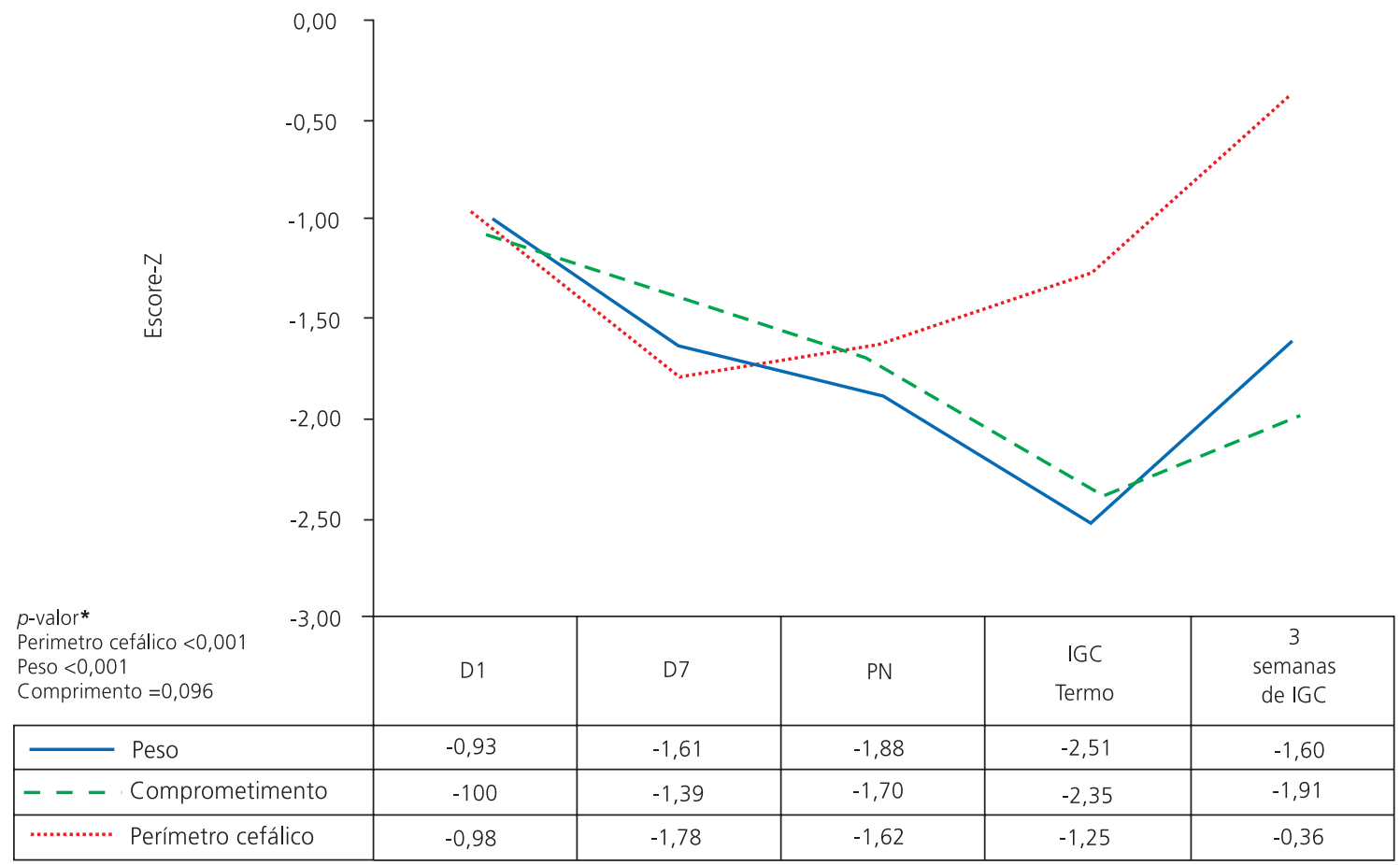

Figura 2. Evolução do escore-Z de peso, comprimento e perímetro cefálico para a idade gestacional dos recém-nascidos pré-termo. Instituto Nacional de Saúde da Mulher, da Criança e do Adolescente Fernandes Figueira, Rio de Janeiro (RJ), 2010.

Nota: *Comparação realizada apenas nos momentos da Idade Gestacional Corrigida (IGC) de termo e três semanas de IGC.

D1: Primeiro dia de vida; D7: Sétimo dia de vida; PN: Dia de recuperação do peso de nascimento.

alcançarem três semanas de idade gestacional corrigida, a circunferência abdominal e a prega cutânea tricipital apresentaram-se significativamente maiores para pesos equivalentes $(p<0,001)$ (Tabela 2).

Ao analisar a razão entre água corporal total (\%) e prega cutânea tricipital, verificou-se que houve uma diferença significativa desta razão entre os RNPT e o grupo de referência, tanto na idade gestacional corrigida de termo como com três semanas de idade gestacional corrigida (Figura 3). Observa-se nos RNPT que, para cada milímetro de prega cutânea tricipital, têm-se 38 de água corporal na idade gestacional corrigida de termo e $14,9 \%$ às três semanas de idade gestacional corrigida. O grupo de referência apresentou a razão entre água corporal total (\%) e prega cutânea tricipital de 19,0\%, resultado que demons- 
Tabela 2. Comparação das medidas antropométricas e da porcentagem de água corporal total dos recém-nascidos a termo com os recém-nascidos pré-termo (na IGC de termo e com 3 semanas de IGC). Instituto Nacional de Saúde da Mulher, da Criança e do Adolescente Fernandes Figueira, Rio de Janeiro (RJ), 2010.

\begin{tabular}{|c|c|c|c|c|c|c|c|c|}
\hline \multirow{3}{*}{ Variáveis } & \multicolumn{4}{|c|}{ Pré-termo } & \multirow{2}{*}{\multicolumn{2}{|c|}{$\begin{array}{l}\text { Termo } \\
(n=32)\end{array}$}} & \multirow{2}{*}{\multicolumn{2}{|c|}{$p$-valor }} \\
\hline & \multicolumn{2}{|c|}{ IGC termo $(n=39)$} & \multicolumn{2}{|c|}{3 semanas de IGC $(n=32)$} & & & & \\
\hline & M & $\mathrm{DP}$ & M & $\mathrm{DP}$ & M & $\mathrm{DP}$ & IGC termo & 3 semanas de IGC \\
\hline Z peso & $-2,51$ & 1,2 & $-1,59$ & 0,9 & $-0,49$ & 0,5 & $<0,001$ & $<0,001$ \\
\hline Z comprimento & $-2,35$ & 1,1 & $-1,91$ & 1,1 & $-0,50$ & 0,6 & $<0,001$ & $<0,001$ \\
\hline $\mathrm{ZPC}$ & $-1,25$ & 1,1 & $-0,36$ & 1,0 & $-0,25$ & 0,7 & $<0,001$ & 0,621 \\
\hline $\mathrm{CA}(\mathrm{cm})$ & 29,70 & 2,6 & 33,50 & 2,3 & 31,60 & 1,7 & 0,001 & $<0,001$ \\
\hline $\mathrm{PCT}(\mathrm{mm})$ & 2,80 & 1,4 & 4,60 & 1,0 & 3,70 & 0,5 & 0,003 & $<0,001$ \\
\hline $\operatorname{ACT}(\%)$ & 70,50 & 3,5 & 65,10 & 1,6 & 68,00 & 2,8 & 0,002 & $<0,001$ \\
\hline
\end{tabular}

Nota: Z peso: escore-Z peso para idade gestacional; Z comprimento: escore-Z comprimento para idade gestacional; Z PC: escore-Z perímetro cefálico para idade gestacional; M: Média; DP: Desvio-Padrão; IGC: Idade Gestacional Corrigida; CA: Circunferência Abdominal; PCT: Prega Cutânea Tricipital; ACT: Água Corporal Total.

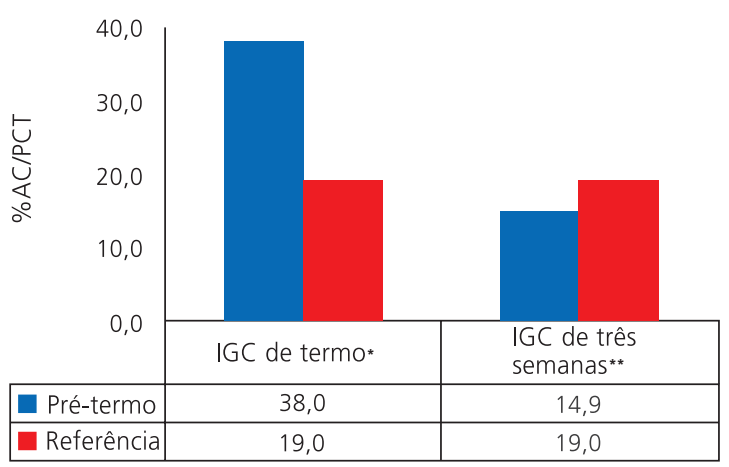

Figura 3. Razão da porcentagem de Água Corporal Total (ACT) e Prega Cutânea Tricipital (PCT) nos recém-nascido prétermo na Idade Gestacional Corrigida (IGC) de termo e com três semanas de IGC. Instituto Nacional de Saúde da Mulher, da Criança e do Adolescente, Fernandes Figueira, Rio de Janeiro (RJ), 2010.

Nota: ${ }^{*} p$-valor $<0,001 ;{ }^{* *} p$-valor $<0,001$

tra que, para um mesmo peso, os RNPT com três semanas de idade gestacional corrigida apresentam menor razão de água corporal total (\%) e prega cutânea tricipital que o grupo de referência.

\section{I S C U S S Ã O}

Este estudo evidencia que muitos RNPT, avaliados com medidas antropométricas e de bioimpedância elétrica, apresentam restrição do crescimento pós-natal e a composição corporal diferente ao alcançarem a idade gestacional corrigida do termo, quando comparados com os que nasceram a termo.

Quanto ao perímetro cefálico, houve recuperação do crescimento mais acelerada após a idade gestacional corrigida de termo. A melhora do escore-Z do perímetro cefálico para idade foi relatada em estudos prévios, principalmente quando comparado com o escore- $Z$ de peso e comprimento 29,30 . Diferentemente do peso e do comprimento, que só apresentaram recuperação após a idade do termo, o crescimento do perímetro cefálico, usado como proxy do crescimento cerebral, iniciou uma recuperação lenta após o sétimo dia de vida, que se acentuou entre a idade gestacional corrigida de termo e três semanas de idade gestacional corrigida. Esse fato talvez indique que o crescimento cerebral esteja preservado. A importância desse achado deve-se ao fato de o crescimento pós-natal do perímetro cefálico indicar um melhor prognóstico em relação ao desenvolvimento cognitivo, apesar da restrição do crescimento pós-natal ${ }^{31}$.

Na comparação realizada quando os RNPT alcançaram o peso que corresponde a 3,0-3,5 kg, verificou-se o aumento da circunferência abdominal e da prega cutânea tricipital assim como uma menor porcentagem de água corporal total em relação aos que nasceram a termo, o que pode 
refletir um aumento na porcentagem da gordura corporal. O estudo de Roggero et al. ${ }^{32}$ também demonstra que ao alcançarem a idade do termo, os RNPT apresentam menor peso, menor comprimento e menor perímetro cefálico e a composição corporal diferente dos que nasceram a termo, sendo maior a porcentagem de gordura corporal total entre os RNPT. Esses achados são semelhantes aos da metanálise publicada por Johnson et al. ${ }^{18} \mathrm{em} 2012$, que também verificaram maior porcentagem de gordura corporal entre os RNPT, principalmente às custas de menor massa livre de gordura. Um aumento da massa de gordura pode estar associado ao desenvolvimento futuro de doenças crônicas não transmissíveis do adulto, como obesidade, diabetes, hipercolesterolemia e hipertensão arterial, que têm sido descritos em recém-nascidos que sofreram restrição de crescimento ${ }^{33}$.

A porcentagem de recém-nascidos com restrição do crescimento pós-natal varia entre os hospitais e depende das práticas nutricionais, dentre outros fatores ${ }^{8}$. Este estudo observou que a restrição do crescimento pós-natal ocorreu em $71 \%$ dos RNPT que apresentaram muito baixo peso ao nascer, enquanto ocorreu em 63 e 39\% nos estudos de Gianini et al. ${ }^{34}$ e de Lima et al. ${ }^{35,36}$. Embleton et al. ${ }^{37}$ demonstraram que a restrição do crescimento pós-natal está relacionada com a deficiência cumulativa da ingestão proteica e calórica observada até a quinta semana de vida. Simon et al. ${ }^{10}$ evidenciaram uma associação entre a baixa idade gestacional e a diminuição do escore-Z do peso durante a internação com um maior risco de déficit de massa livre de gordura. Apesar de as avaliações terem sido realizadas em momentos diferentes (alta hospitalar no estudo de Simon et al. ${ }^{10}$ e na idade gestacional corrigida de termo no nosso estudo), os resultados foram semelhantes, assim como os resultados do estudo de Daly-Wolfe et al. ${ }^{38}$.

O crescimento pós-natal adequado dos RNPT continua sendo um desafio na neonatologia. A meta é ainda tentar alcançar extraútero a velocidade de crescimento da vida fetal, preser- vando principalmente o crescimento cerebral|4-6,39. Por outro lado, um crescimento acelerado após um período de restrição, fenômeno intitulado como catch-up, aumenta a preocupação com a síndrome metabólica no futuro. Existe sim uma relação entre o catch-up e o desenvolvimento dos RNPT, mas o momento em que a recuperação do crescimento deve ocorrer ainda não está estabelecido ${ }^{40}$.

O estudo evidenciou a recuperação do peso entre a idade gestacional corrigida de termo e três semanas de idade gestacional corrigida, principalmente com predomínio de gordura. A maioria dos recém-nascidos desta coorte apresentou restrição do crescimento na idade gestacional corrigida do termo e semelhante aos achados de Roggero et al. ${ }^{41}$ : tanto a restrição de crescimento intrauterino como a pós-natal influenciam a velocidade de recuperação da massa de gordura corporal. Nesse estudo citado, os RNPT foram divididos em três grupos, e os que nasceram pequenos para idade gestacional e os que apresentaram restrição do crescimento pós-natal apresentaram um ganho maior de massa de gordura que os sem restrição do crescimento pós-natal. No período pós-natal imediato, os RNPT acumulam mais gordura que os a termo e apresentam a recuperação da massa de gordura mais precocemente que a recuperação do peso e comprimento ${ }^{33}$.

Um melhor entendimento sobre o crescimento pós-natal dos RNPT, durante a internação e após a alta hospitalar, torna-se necessário devido às implicações na saúde futura desses recém-nascidos ${ }^{42}$. Futuros estudos que incorporem informações nutricionais com o perfil de ganho de peso, perímetro cefálico, comprimento, massa livre de gordura e massa de gordura são importantes para determinação da melhor forma de nutrir esses recém-nascidos, proporcionando um melhor crescimento e desenvolvimento sem aumentar o risco de doença metabólica no futuro.

Uma das limitações deste estudo foi não registrar variáveis nutricionais longitudinalmente nos mesmos momentos das avaliações antropométricas e de bioimpedância. O seguimento dos 
recém-nascidos até a idade pré-escolar também seria interessante para permitir a análise de fatores que poderiam estar associados à incidência de sobrepeso e obesidade, ainda com poucos estudos a respeito ${ }^{43}$. Além disso, a bioimpedância elétrica nessa faixa etária não permite a avaliação da massa livre de gordura e massa de gordura, o que torna necessários futuros estudos que utilizem métodos e técnicas que permitam essa avaliação.

A curva usada para determinação do escore-Z para peso, comprimento e perímetro cefálico de Fenton \& Kim ${ }^{28}$ também fornece dados transversais do momento de nascimento, refletindo a situação nutricional ao nascer, semelhante à curva de Olsen et al..$^{44}$ e à curva do estudo de Niklasson et al. ${ }^{45}$.

\section{O N CLUS Ã O}

Os recém-nascidos pré-termo apresentam perfil antropométrico e de água corporal diferente dos recém-nascidos a termo, sugerindo acúmulo de gordura. A recuperação do crescimento mais evidente em relação ao perímetro cefálico é um achado importante, pois talvez indique que o crescimento cerebral esteja preservado. Novas pesquisas na área são necessárias para melhor entendimento sobre como esses recém-nascidos crescem fora do útero, quais suas reais necessidades nutricionais durante a internação e após a alta hospitalar e como essa situação influencia o desenvolvimento neuropsicomotor.

\section{COLABORADORES}

LD VILLELA contribuiu para elaboração do projeto, coleta de dados, análise e interpretação dos dados, elaboração do artigo e aprovou a versão final do artigo. FVM SOARES, AD ABRANCHES, SC GOMES JÚNIOR, MDBB MÉIO, MEL MOREIRA contribuíram para elaboração do projeto, análise e interpretação dos dados, elaboração do artigo e aprovaram a versão final do artigo.

\section{REFERÊ NCIAS}

1. Uauy R, Koletzko B. Defining the nutritional needs of preterm infants. In: Koletzko B, Poindexter B, Uauy R. Nutritional care of preterm infants: Scientific basis and practical guidelines. World Review of Nutrition and Dietetics. Basel $(\mathrm{CH})$ : Karger; 2014.

2. Belfort MB, Gillman MW, Buka SL, Casey PH, McCormick MC. Preterm infant linear growth and adiposity gain: Trade-offs for later weight status and intelligence quotient. J Pediatr. 2013; 163(6):1564-9. http://dx.doi.org/10.1016/j.jpeds. 2013.06.032

3. Ehrenkranz RA, Dusick AM, Vohr BR, Wright LL, Wrage LA, Poole WK. Growth in neonatal intensive care unit influences neurodevelopmental and growth outcomes of extremely low birth weight infants. Pediatrics. 2006; 117(4):1253-61. http:// dx.doi.org/10.1542/peds.2005-1368

4. American Academy of Pediatrics Committee on Nutrition. Nutritional needs of low-birth-weight infants. Pediatrics. 1985; 75(5):976-86.

5. Agostoni C, Buonocore G, Carnielli VP, De Curtis $M$, Darmaun D, Decsi T, et al. Enteral nutrient supply for preterm infants: Commentary from the European Society for Paediatric Gastroenterology, Hepatology, and Nutrition Committee on Nutrition. J Pediatr Gastroenterol Nutr. 2010; 50(1):85-91. http://dx.doi.org/10.1097/MPG.0b013e3181a daee 0

6. Embleton ND, Simmer K. Practice of parenteral nutrition in VLBW and ELBW infants. In: Koletzko $B$, Poindexter B, Uauy R. Nutritional care of preterm infants: Scientific basis and practical guidelines. World Review of Nutrition and Dietetics. Switzerland: Karger; 2014.

7. Ehrenkranz RA, Younes N, Lemons JA, Fanaroff AA, Donovan $\mathrm{EF}$, Wright $\mathrm{LL}$, et al. Longitudinal growth of hospitalized very low birth weight infants. Pediatrics. 1999; 104(2 Pt. 1):280-9.

8. Horbar JD, Ehrenkranz RA, Badger GJ, Edwards EM, Morrow KA, Soll RF, et al. Weight growth velocity and postnatal growth failure in infants 501 to 1500 grams: 2000-2013. Pediatrics. 2015; 136(1):e84-92. http://dx.doi.org/10.1542/peds.2015-0129

9. Ehrenkranz RA. Early, aggressive nutricional management for very low birth weight infants: What is the evidence? Semin Perinatol. 2007; 31(2):48-55. http://dx.doi.org/10.1053/j.semperi. 2007.02.001

10. Simon L, Frondas-Chauty A, Senterre T, Flamant $C$, Darmaun D, Rozé JC. Determinants of body composition in preterm infants at the time of hospital discharge. Am J Clin Nutr. 2014; 100(1):98-104. http://dx.doi.org/10.3945/ajcn.113.080945 
11. Martin CR, Brown YF, Ehrenkranz RA, O'Shea TM, Allred EN, Belfort MB, et al. Nutritional practices and growth velocity in the first month of life in extremely premature infants. Pediatrics. 2009; 124(2):649-57. http://dx.doi.org/10.1542/peds. 2008-3258

12. Mathai S, Derraik JGB, Cutfield WS, Dalziel SR, Harding JE, Biggs J, et al. Increased adiposity in adults born preterm and their children. PLOS ONE. 2013; 8(11):e81840. http://dx.doi.org/10.1371/ journal.pone.0081840

13. Leunissen RWJ, Kerkhof GF, Stijnen T, HokkenKoelega ACS. Effect of birth size and catch-up growth on adult blood pressure and carotid intimamedia thickness. Horm Res Paediatr. 2012; 77(6):394-401. http://dx.doi.org/10.1159/0003 38791

14. Kerkhof GF, Hokken-Koelega ACS. Rate of neonatal weight gain and effects on adult metabolic health. Nat Rev Endocrinol. 2012; 8(11):689-92. http://dx. doi.org/10.1038/nrendo.2012.168

15. Modi N, Thomas EL, Harrington TAM, Uthaya S, Doré CJ, Bell JDB. Determinants of adiposity during preweaning postnatal growth in appropriately growth and growth-restricted term infants. Pediatr Res. 2006; 60(3):345-8. http://dx.doi.org/10.1203/ 01.pdr.0000232732.93000.52

16. Uthaya S, Thomas EL, Hamilton G, Doré CJ, Bell J, Modi N. Altered adiposity after extremely preterm birth. Pediatr Res. 2005; 57(2):211-5. http:// dx.doi.org/10.1203/01.PDR.0000148284.58 934.1C

17. Barker DJ. The origins of the developmental origins theory. J Intern Med. 2007; 261(5):412-7. http:// dx.doi.org/10.1111/j.1365-2796.2007.01809.x

18. Johnson MJ, Wootton SA, Leaf AA, Jackson AA. Preterm birth and body composition at term equivalent age: A systematic review and metaanalysis. Pediatrics. 2012; 130(3):e640-9. http:// dx.doi.org/10.1542/peds.2011-3379

19. Ballard JL, Khoury JC, Wedig K, Wang L, EilersWalsman BL, Lipp R. New Ballard score, expanded to include extremely premature infants. J Pediatr. 1991; 119(3):417-23.

20. Rodríguez G, Ventura P, Samper MP, Moreno L, Sarría A, Pérez-González JM. Changes in body composition during the initial hours of life in breastfed healthy term newborns. Biol Neonate. 2000; 77(1):12-6.

21. Villela LV. O crescimento e a composição corporal dos recém-nascidos prematuros de muito baixo peso ao nascer [dissertação]. Rio de Janeiro: Fiocruz; 2007.

22. Méio MD, Sichieri R, Soares FV, Moreira ME. Total body water in small- and appropriate- for gestational age newborns. J Perinat Med. 2008; 36(4):354-8. http//:dx.doi.org/10.1515/JPM.2008. 058

23. Tang W, Ridout D, Modi N. Assessment of total body water using bioelectrical impedance analysis in neonates receiving intensive care. Arch Dis Child Fetal Neonatal. 1997; 77(2):123-6. http://dx.doi. org/10.1136/fn.77.2.F123

24. Moreira MEL. Avaliação do crescimento e composição corporal de recém-nascidos pré-termo [doutorado]. São Paulo: Universidade de São Paulo; 1997.

25. Sasanow SR, Georgieff MK, Pereira GR. Mid-arm circumference and mid-arm/head circumference ratios: Standard curves for anthropometric assessment of neonatal nutritional status. J Pediatr. 1986; 109(2):311-5.

26. Sociedade Brasileira de Pediatria, Departamento Científico de Nutrologia. Avaliação nutricional da criança e do adolescente: manual de orientação. Rio de Janeiro: Sociedade Brasileira de Pediatria; 2009.

27. Catalano PM, Drago NM, Amini SB. Factors affecting fetal growth and body composition. Am J Obstet Gynecol. 1995; 172(5):1459-63. http:// dx.doi.org/10.1016/0002-9378(95)90478-6

28. Fenton TR, Kim JH. A systematic review and metaanalysis to revise the Fenton growth chart for preterm infants. BMC Pediatrics. 2013; 13:59. http:// dx.doi.org/10.1186/1471-2431-13-59

29. Cooke RJ, Griffin I. Altered body composition in preterm infants at hospital discharge. Acta Paediatr. 2009; 98(8):1269-73. http://dx.doi.org/10.1111/j. 1651-2227.2009.01354.x

30. Costa-Orvay JA, Figueras-Aloy J, Romera G, ClosaMonasterolo R, Carbonell-Estrany $X$. The effects of varing protein and energy intakes on the growth and body composition of very low birth weight infants. Nutr J. 2011; 10:140. http://dx.doi.org/ 10.1186/1475-2891-10-140

31. Cheong JLY, Hunt RW, Anderson PJ, Howard K, Thompson DK, Wang HX, et al. Head growth in preterm infants: Correlation with magnetic resonance imaging and neurodevelopmental outcome. Pediatrics. 2008; 121(6):e1534-40. http:// dx.doi.org/10.1542/peds.2007-2671

32. Roggero $P$, Giannì ML, Amato $O$, Orsi A, Piemontese $P$, Morlacchi $L$, et al. Is term newborn body composition being achieved postnatally in preterm infants? Early Hum Dev. 2009; 85(6):349-52. http:// dx.doi.org/10.1016/j.earlhumdev.2008.12.011

33. Okada T, Takahashi S, Nagano N, Yoshikawa K, Usukura $Y$, Hosono S. Early postnatal alteration of body composition in preterm and small-for- 
gestational-age infants: Implications of catch-up fat. Pediatr Res. 2015; 77(1-2):136-42. http://dx. doi.org/10.1038/pr.2014.164

34. Gianini NM, Vieira AA, Moreira MEL. Avaliação dos fatores associados ao estado nutricional na idade corrigida de termo em recém-nascidos de muito baixo peso. J Pediatr. 2005; 81(1):34-40.

35. Lima PA, Carvalho M, Costa AC, Moreira ME. Variables associated with extra uterine growth restriction in very low birth weight infants. J Pediatr. 2014; 90(1):22-7. http://dx.doi.org/10.1016/j.jped. 2013.05.007

36. Lima PA, de Carvalho M, da Costa AC, Moreira ME. Author's reply: Z-Score: Fenton 2013. Ten-year update. J Pediatr. 2014; 90(4):427-8. http://dx.doi. org/10.1016/j.jped.2014.04.004

37. Embleton NE, Pang N, Cooke RJ. Postnatal malnutrition and growth retardation: An inevitable consequense of current recommendations in preterm infants? Pediatrics. 2001; 107(2):270-3. http://dx.doi.org/10.1542/peds.107.2.270

38. Daly-Wolfe K, Jordan K, Slater H, Beachy J, MoyerMileur L. Mid-arm circumference is a reliable predictor of adiposity in preterm and term infants. Pediatr Res. 2015; 78(3):336-41. http://dx.doi.org/ 10.1038/pr.2015.103.

39. Rice MS, Valentine CJ. Neonatal body composition: Measuring lean mass as a tool to guide nutrition management in the neonate. Nutr Clin Pract. 2015;30(5):625-32. http://dx.doi.org/10.1177/08 84533615578917
40. Cooke R. Nutrition of preterm infants after discharge. Ann Nutr Metab. 2011; 58(Suppl. 1):32-6. http:// dx.doi.org/10.1159/000323385

41. Roggero $P$, Giannì ML, Liotto N, Taroni F, Orsi A, Amato $\mathrm{O}$, et al. Rapid recovery of fat mass in small for gestational age preterm infants after term. PLoS ONE. 2011; 6(1):e14489. http://dx.doi.org/10.13 71/journal.pone.0014489

42. Giannì ML, Roggero $P$, Piemontese $P$, Orsi $A$, Amato O, Taroni $\mathrm{F}$, et al. Body composition in newborn infants: 5-year experience in an Italian neonatal intensive care unit. Early Hum Dev. 2012; 88(Suppl. 1):13-7. http://dx.doi.org/10.1016/j.earlhumdev. 2011.12.022

43. Menezes CFB, Neves J, Gonsalez PS, Vasconcelos FAG. Incidência de sobrepeso/obesidade em pré-escolares durante dois anos de acompanhamento. Rev Nutr. 2014; 27(3):269-78. http://dx.doi.org/ 10.1590/1415-52732014000300001

44. Olsen IE, Lawson ML, Ferguson AN, Cantrell R, Grabich SC, Zemel BS, et al. BMI curves for preterm infants. Pediatrics. 2015; 135(3):e572-81. http:// dx.doi.org/10.1542/peds.2014-2777

45. Niklasson A1, Albertsson-Wikland K. Continuous growth reference from $24^{\text {th }}$ week of gestation to 24 months by gender. BMC Pediatr. 2008; 8:8. http://dx.doi.org/10.1186/1471-2431-8-8

Recebido: junho 26, 2014 Versão final: julho 13, 2015 Aprovado: agosto 4, 2015 
